\title{
Phenotypic characteristics of colorectal cancer in BRCA1/2 mutation carriers
}

\author{
Albert Grinshpun ${ }^{1} \cdot$ Naama Halpern ${ }^{2,5} \cdot$ Roy Zvi Granit $\mathbb{D}^{3} \cdot$ Ayala Hubert $^{1} \cdot$ Tamar Hamburger $^{1} \cdot$ Yael Laitman $^{4,5}$. \\ Einat Shacham-Shmueli ${ }^{2,5} \cdot$ Yehudit Peerless $^{1} \cdot$ Eitan Friedman $^{4,5} \cdot$ Tamar Peretz $^{1}$
}

Received: 22 March 2017 / Revised: 9 September 2017 / Accepted: 23 November 2017 / Published online: 10 January 2018

(c) European Society of Human Genetics 2018

\begin{abstract}
Mutations in the BRCA1/2 genes were recently shown to be associated with an increased risk for colorectal cancer. We characterized the largest cohort available of BRCA1/2 mutation carriers with colorectal cancer. We analyzed 32 patients with lower gastrointestinal cancers and germline BRCA1/2 mutations from two large academic hospital registries; $91 \%$ of patients were of Ashkenazi ancestry, $78 \%$ were women, and $62.5 \%$ were carriers of BRCA1 gene mutations. A high percentage of colorectal tumors $(34.5 \%)$ had a mucinous histology and were located atypically in the left colon. Two patients had anal cancer with unusual histology and an additional patient had mucinous small bowel carcinoma. Gene expression analysis showed significant correlation between the gene signatures of left mucinous colorectal cancer and basal-like breast cancer. Our results imply that Ashkenazi BRCA1/2 mutation carriers with colorectal cancer might have unique characteristics with a high rate of left-sided, mucinous histology colorectal cancer, and possibly anal carcinoma. This report suggests a phenotypic influence of defects in DNA repair genes on colorectal tumors.
\end{abstract}

\section{Introduction}

BRCA1/2 genes are responsible for numerous cellular functions, primarily the maintenance of genome integrity and response to DNA damage [1]. Deleterious mutations in BRCA1/2 genes cause a highly penetrant, hereditary breast, and ovarian cancer syndrome. Although these genes are damaged in every cell of a carrier's body, the

Electronic supplementary material The online version of this article (https://doi.org/10.1038/s41431-017-0067-1) contains supplementary material, which is available to authorized users.

Albert Grinshpun

Albertg@hadassah.org.il

1 Sharett Institute of Oncology, Hadassah-Hebrew University Medical Center, Jerusalem, Israel

2 Institute of Oncology, Chaim Sheba Medical Center, Tel Hashomer, Ramat Gan, Israel

3 Department of Developmental Biology and Cancer Research, Institute for Medical Research Israel-Canada, The Hebrew University-Hadassah Medical School, Jerusalem, Israel

4 Susanne Levy Gertner Oncogenetics Unit, Chaim Sheba Medical Center, Tel Hashomer, Ramat Gan, Israel

5 Sackler Faculty of Medicine, Tel Aviv University, Tel Aviv, Israel tissue specificity of tumor development is still unknown. There are conflicting data in the literature regarding an increased prevalence for colorectal cancer (CRC) in BRCA1/2 mutation carriers [2-5]. However, a recent prospective trial provided strong evidence in favor of a true increased risk of CRC [6]. This trial followed 7000 BRCA1/2 mutation carriers and showed increased risk for CRC in female carriers of the BRCA1 gene mutation who were under the age of 50. Therefore, screening was recommended for this population from the age of 40. Given the lack of data regarding the nature, pathology, and clinical features of intestinal cancers in BRCA1/2 mutation carriers, we collected all available cases from two large academic medical centers. In this study, we characterized the largest cohort of lower alimentary tract cancers in BRCA $1 / 2$ mutation carriers and shed light on these clinical entities.

\section{Methods}

We used two large academic hospitals' registries, the Hadassah Medical Center and Sheba Medical center genetic registries, which together contain more than $3000 \mathrm{BRCA} 1 / 2$ mutation carriers. We found 44 cases of patients with 
intestinal (small and large bowel) or anal cancer. Clinicopathological data were found in computerized and archived records and were available for 32 of these patients $(32 / 44=$ $73 \%$ ). All research was approved by the local ethics committees.

For gene set correlations, level 3 expression and clinical data (primarily sidedness) were obtained from The Cancer Genome Atlas (TCGA) Network website [7]. The data were then normalized to the mean of each gene. The Spearman correlation was calculated using Matlab (MathWorks Inc.) comparing each sample to either the basal-like breast cancer PAM50 vector [8] or the complete basal-like signature [9] to obtain the $\mathrm{R}$ value. The latter signature was processed as previously described [10].

For statistical analysis, Fisher's exact test and Student's $t$ test were used; $P$-values were two-tailed and significant when $P$ was $<0.05$. The binomial test was performed with $\mathrm{R}$ software (www.r-project.org) and the Kaplan-Meier log rank test was used to assess significance.

\section{Results}

Thirty-two patients were eligible for the study (Table 1), $\sim 1 \%$ of our total cohort of BRCA1/2 mutation carriers. Most patients were of Ashkenazi ancestry $(29,91 \%)$. The majority of them were women $(25 / 32,78 \%)$ and carriers of BRCA1 gene mutations $(20 / 32,62.5 \%)$. The most common mutations were the Ashkenazi founder mutations: c.68_69delAG and c.5266dupC (formerly 185delAG and 5382insC, respectively) in the BRCA1 gene and c.5946delT (classically 6174delT) in BRCA2 (additional details are found in Supplementary Table 1). Fourteen patients were also screened for the APC c.3920 T > A (p.I1307K) mutation, and two (14\%) of these patients were positive.

Seventy-two percent of the women (18 patients) had breast cancer, most of them occurring prior to the intestinal cancer diagnosis. Male carriers with breast cancer were not found in our cohort. In addition, seven women had ovarian cancer before the colorectal tumor diagnosis. The average ages $( \pm \mathrm{SD})$ at diagnosis were $54.7 \pm 14$ and $57.7 \pm 8.1$ years for the first breast and ovarian cancer, respectively. The age of the first breast cancer diagnosis in our cohort is relatively older than the average of the Hadassah Medical Center database $(44.14 \pm 10.9$ years for female BRCA1/2 carriers, $n=566, P<0.05$ Student's $t$-test). Six women (24\%) underwent prophylactic total abdominal hysterectomy and bilateral salpingo-oophorectomy before intestinal cancer was detected.

Colorectal tumor characteristics are described in Table 2. The tumor location was not classically distributed [11] and we observed a mild "left shift" as $27 \%$ were in the right colon and $73 \%$ were in the left colon, including the rectum.
Table 1 Patients' characteristics $(n=32)$

\begin{tabular}{|c|c|}
\hline \multicolumn{2}{|l|}{ Gender } \\
\hline Male & 7 \\
\hline Female & 25 \\
\hline \multicolumn{2}{|l|}{ BRCA1 gene mutations $(n=20)$} \\
\hline c.68_69delAG & 16 \\
\hline c.5266dupC & 2 \\
\hline c. $5123 \mathrm{C}>\mathrm{A}$ & 2 \\
\hline \multicolumn{2}{|l|}{ BRCA2 gene mutations $(n=12)$} \\
\hline c.5946delT & 11 \\
\hline c.3847_3848delGT & 1 \\
\hline $\begin{array}{l}\text { Age at diagnosis of intestinal cancer } \\
\text { (average years } \pm S D \text {, range) }\end{array}$ & $60.5 \pm 12.1(31-85)$ \\
\hline BRCA1 $(n=19) / \operatorname{BRCA} 2(n=10)$ & $60.7 \pm 10 / 58.6 \pm 16.4$ \\
\hline $\begin{array}{l}\text { Age at diagnosis of anal tumor (average } \\
\text { years } \pm S D \text {, range) }\end{array}$ & $41 \pm 11.3(33-49)$ \\
\hline Personal history of breast cancer $(n, \%)$ & $18,72 \%$ \\
\hline $\begin{array}{l}\text { Age at first breast cancer diagnosis } \\
\text { (average years } \pm \mathrm{SD} \text {, range) }\end{array}$ & $54.7 \pm 14(33-77)$ \\
\hline BRCA1 $(n=12) /$ BRCA2 $(n=6)$ & $52.3 \pm 14.7 / 59.7 \pm 12.2$ \\
\hline Personal history of ovarian cancer $(n, \%)$ & $7,28 \%$ \\
\hline $\begin{array}{l}\text { Age at ovarian cancer diagnosis (average } \\
\text { years } \pm S D \text {, range) }\end{array}$ & $57.7 \pm 7.3(51-69)$ \\
\hline BRCA1 $(n=5) /$ BRCA2 $(n=2)$ & $59.2 \pm 8.1 / 54 \pm 4.2$ \\
\hline
\end{tabular}

The average ages $( \pm \mathrm{SD})$ at diagnosis were $59.5 \pm 12.9$ and $62.6 \pm 10.9$ years, for the left and right colon, respectively. The age at diagnosis did not differ significantly between BRCA1 to BRCA2 mutation carriers. In addition to CRC, two male patients (aged 49 and 33 years) had anal cancer (carriers of BRCA1 c.68_69delAG and BRCA2 c.5946delT), with an unusual histology: adenosquamous carcinoma and adenocarcinoma with neuroendocrine features. Both were negative for HIV infection. An additional female patient (aged 65 years) had small bowel mucinous adenocarcinoma with a personal and family history of juvenile polyposis. As described previously, right-sided colon cancers are more common in women in the general population [12]. In our cohort, all right-sided tumors were found in women (9/9). Interestingly, a high percentage of patients (eight women and two men, 34.5\% of colorectal tumors) had mucinous histology tumors that were located atypically in the left colon. This high proportion is statistically significant compared with the expected incidence [13] of $15 \%$ ( $P=0.007,95 \%$ CI 17.9-54.3, Binomial test). The average age $( \pm \mathrm{SD})$ at the diagnosis of a mucinous colorectal tumor was $63.4 \pm 13.8$ years (range: $41-85$ ) and $80 \%$ of these patients harbored a BRCA1 gene mutation (non-significant). Furthermore, the majority of the ovarian cancer patients developed colorectal tumors with mucinous histology $(5 / 7,71 \%)$, at a median of 6 years later (range 
Table 2 Colorectal tumors' characteristics $(n=29)$

\begin{tabular}{|c|c|c|}
\hline Location & Right $(n=9)$ & Left $(n=20)$ \\
\hline Cecum & 6 & 0 \\
\hline Ascending colon/hepatic flexure & 3 & 0 \\
\hline $\begin{array}{l}\text { Descending colon/splenic } \\
\text { flexure }\end{array}$ & 0 & 1 \\
\hline Sigmoid & 0 & 9 \\
\hline Rectosigmoid & 0 & 3 \\
\hline Rectum & 0 & 7 \\
\hline \multicolumn{3}{|l|}{ Stage of colorectal tumors } \\
\hline 0 & 0 & 1 \\
\hline I & 1 & 3 \\
\hline II & 4 & 5 \\
\hline III & 2 & 6 \\
\hline IV & 2 & 5 \\
\hline \multicolumn{3}{|l|}{ Differentiation } \\
\hline Well & 3 & 2 \\
\hline Moderately & 3 & 7 \\
\hline Poor & 0 & 0 \\
\hline Mucinous & 2 & 8 \\
\hline Not determined & 1 & 3 \\
\hline \multirow[t]{2}{*}{$\begin{array}{l}\text { Age at diagnosis (average years } \\
\pm \mathrm{SD} \text {, range) }\end{array}$} & $\begin{array}{l}59.5 \pm 12.9 \\
(41-75)\end{array}$ & $\begin{array}{l}62.6 \pm 10.9 \\
(31-85)\end{array}$ \\
\hline & Women only & \\
\hline $\begin{array}{l}\text { Fraction of BRCA1 mutation } \\
\text { carriers }\end{array}$ & $67 \%$ & $70 \%$ \\
\hline
\end{tabular}

1-19). The percentage of these patients was nearly significant compared with those who developed a nonmucinous histology adenocarcinoma $(P=0.07$, Fisher's Exact test). Data about non-surgical treatment modalities (platinum chemotherapy administration, etc.) were unavailable for most patients due to archival data retrieval difficulties.

In our cohort, two patients had additional non-typical tumors, Hodgkin's lymphoma and cervical squamous cell carcinoma, before the CRC diagnosis. Another three patients had synchronous tumors. Two tumors were found by chance during the patient's colectomy (gall bladder adenocarcinoma and appendiceal carcinoid). The third cancer was a mucosa-limited gastric adenocarcinoma, which was synchronously detected and resected during the removal of colon cancer.

During our follow-up (2000-2016), 12 patients died. Seven patients $(21.9 \%)$ presumably died from their CRC (stages III and IV only at diagnosis), and three of them had the mucinous adenocarcinoma subtype (one of them originated in right colon). To examine the relationship between mucinous colorectal tumors (mainly in the left colon) and BRCA1/2-related tumors on the molecular level, we performed gene expression analysis on published TCGA data
[7]. We compared the expression profiles of mucinous colorectal tumors with the known gene signatures of BRCA1/2-related tumors: the homologous recombination repair deficiency (HRD) [14], BRCAness [15], and basallike breast cancer signatures [8, 9]. Two basal-like signatures were used, a limited 50 gene set (well established PAM50 signature) and extensive 1400 gene set comprising the complete signature. There were no correlations with the HRD or BRCAness signatures (data not shown). Yet, left mucinous tumors were significantly correlated with the basal-like breast cancer signatures (Fig. 1a, b) when dividing TCGA data according to sidedness (left/right) and histology (adenocarcinoma/mucinous). Using the same anatomic and pathological subgroups, we compared the survival Kaplan-Meier plots from the TCGA data (Fig. 1c) and demonstrated that mucinous tumors have different outcomes depending on the primary tumor location.

\section{Discussion}

CRC is a very common cause of morbidity and mortality in Western countries [16]. There are reports that the incidence of CRC in Ashkenazi Jews is higher than in the general population [17]. This might be owing to multiple ethnogenetic causes that have enriched this population with multiple founder mutations in cancer susceptibility genes, such as BRCA1, BRCA2, MSH2, MSH6, etc. Additional studies have reported an increased incidence of CRC in BRCA1/2 mutation carriers [2, 6].

To the best of our knowledge, this report is the largest cohort of BRCA1/2 carriers who developed CRC. We found that CRC was diagnosed at an earlier age than in Western populations, but the stage distribution was similar [16]. Tumors in our cohort were more often located in the left colon [16, 18]. Generally, women have more right-sided tumors than men [19], as female sex hormones have a protective role [20]. In our cohort, most of the women had breast cancer prior to the CRC diagnosis; whereas, an additional group had ovarian cancer or prophylactic oophorectomy. Taken together, a significant portion of women were exposed to cytotoxic DNA damaging drugs, radiation, estrogen depleting therapy, or simply premature menopause. Furthermore, five out of seven women with ovarian cancer developed mucinous CRC. This may suggest that prior exposure to DNA damaging agents (such as platinum compounds) and low levels of estrogen may affect the genomes of the developing colorectal tumors, favoring a mucinous histology. Thus, it is of great importance to explore the role of treatment with platinum compounds (such as Oxaliplatin) in this group in the future.

Moreover, we found a significantly higher than expected ratio $(34.5 \%)$ of mucinous colorectal adenocarcinomas, 

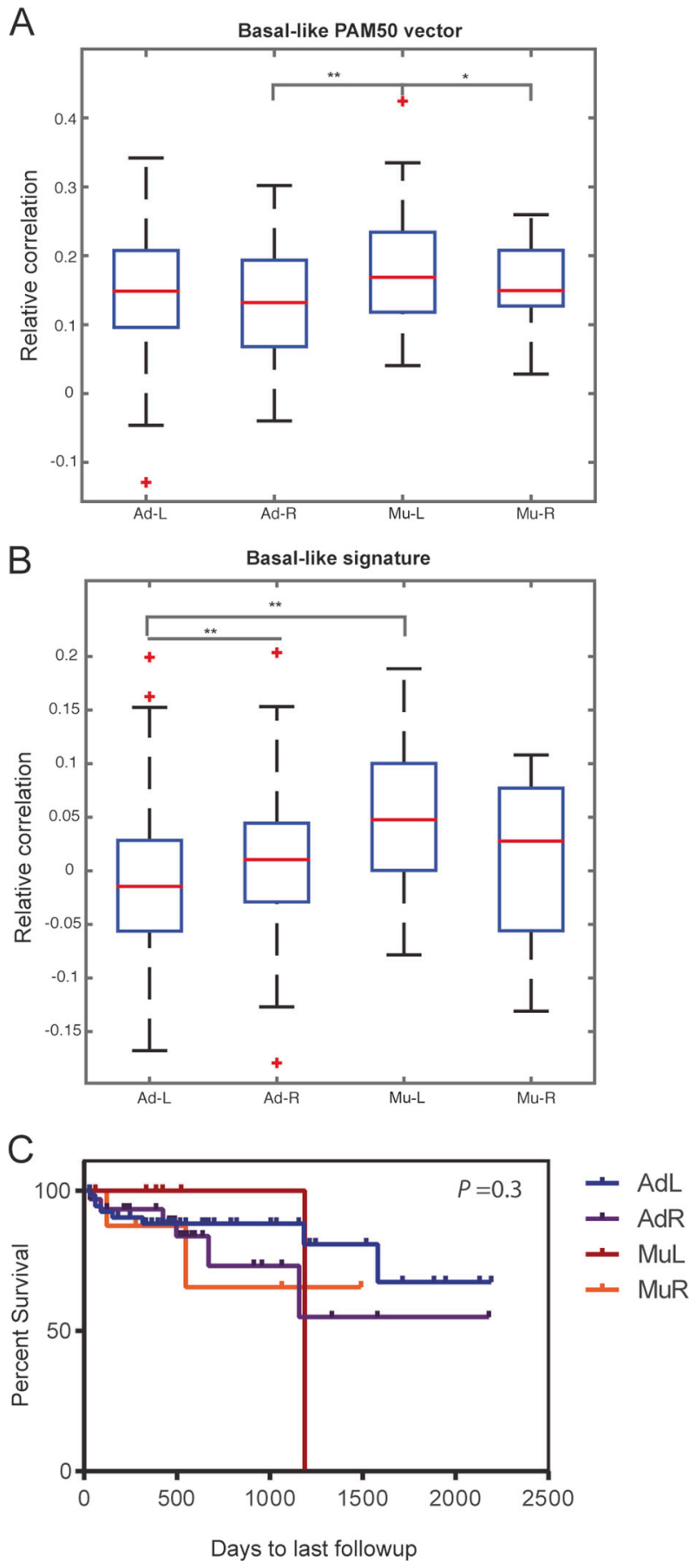

Fig. 1 Correlations with molecular signatures and the survival of right/ left colorectal mucinous or non-mucinous adenocarcinoma tumors. a Boxplot showing the relative correlation of CRC types with the basallike breast cancer PAM50 vector. Each box represents the second and third quartiles within each group; the center line marks the median and plus signs represent outliers. b Same analysis for the complete basallike signature. c Kaplan-Meier plots depicting the survival distribution of the four tumor types. For Kaplan-Meier plots, the log rank test was used to assess significance. $* P<0.05, * * P<0.005$, Student's $t$-test. Ad- $\mathrm{L}=$ left-side adenocarcinoma $(n=114)$, Ad- $\mathrm{R}=$ right-side adenocarcinoma $(n=71)$, Mu-L $=$ left-side mucinous adenocarcinoma $(n=14), \mathrm{Mu}-\mathrm{R}=$ right-side mucinous adenocarcinoma $(n=19)$ mainly in the left colon. The typical presentation of this histologic subtype is in the proximal (right) colon, appearing in up to $15 \%$ of sporadic colorectal cases [13]. Mucinous tumors are linked to Western societies, female sex, abdominal/pelvic radiation, chronic colitis, microsatellite instability, and a worse prognosis [13]. Our analysis revealed that the genetic signature of left mucinous colorectal tumors might best correlate with the basal-like breast tumor molecular signature. In turn, basal-like tumors are closely interlinked to BRCA1/2 germline mutations [1] and their expression profile might represent the BRCA1/2-related tumor signature. As previously mentioned, the lack of correlation with HRD/BRCAness signatures suggests other mechanisms of tumorigenesis. A major drawback in large data sets (such as TCGA) is the low frequency of germline BRCA1/2 mutation carriers among the general study populations; therefore, the molecular portraits of these unique tumors are scarce [18]. Alternatively, this correlation could be explained by the similar dedifferentiation status of both basal-like breast tumors and colorectal mucinous cancers. Specific trials to assess gene expression in various BRCA1/2-related cancers should be undertaken in the future.

There are several indirect lines of evidence in the literature implying there are DNA damage defects in mucinous colorectal tumors. First, Topoisomerase I is overexpressed in these tumors, possibly owing to an impaired DNA repair mechanism [21]. Second, mucinous tumors were mainly classified as Consensus Molecular Subtype 1, which is comprised of hypermutated tumors with defects in DNA repair genes (MSH6, ATM, etc.) [22]. The third clue was shown by Ciriello and coworkers [23], who characterized a subset of ultra-mutated CRC with an altered double-strand brake repair mechanism. Notably, $>50 \%$ of these tumors had somatic mutations in BRCA1/2 genes. One may speculate that the presence of germline defects in DNA repair mechanisms (homologous recombination, mismatch repair etc.), such as mutated BRCA1/2 genes, may shift the developing colorectal tumor pathway towards a mucinous phenotype. Our findings suggest that left-sided mucinous CRC is a new entity with a different etiology and prognosis, possibly related to a germline BRCA1/2 mutation. Perhaps this phenomenon was previously overlooked owing to the low number of germline BRCA1/2 mutations in large, well-described cohorts.

The higher than expected age of breast cancer diagnosis (54.7 years) in our population may be simply owing to bias from the small sample size or attributed to the phenomenon that the age of the onset of breast cancer has decreased over the years (many women in our cohort were diagnosed with breast cancer in the 1980's) [24].

Anal cancer is a rare disease, comprising of only $0.2 \%$ of all cancer types [25]. In our cohort, there were two cases in 
male carriers, one had an adenosquamous histology and the other had a mixed adeno-neuroendocrine carcinoma. Two cases are unexpected in a relatively small cohort. Phelan and coworkers [6], prospectively followed over 7000 women in several countries with BRCA1/2 mutations; they found an increased incidence of CRC and four cases of anal cancer (three with a squamous histology, the other unknown), which led the authors to recommend screening for anal cancer in BRCA1 mutation female carriers aged $\geq 40$ years. These findings and our data suggest that BRCA1/2 mutations are involved in the pathogenesis of anal cancer. Therefore, we suggest screening male BRCA1/ 2 mutation carriers in addition to female carriers, possibly starting under the age of 40 . In conclusion, BRCA1/2 mutation carriers may be prone to developing left-sided mucinous histology CRC and anal carcinoma. Further studies are needed to provide detailed descriptions of these clinico-pathological entities and a better understanding of the molecular mechanisms behind them.

\section{Compliance with ethical standards}

Conflict of interest The authors declare that they have no conflict of interest.

\section{References}

1. Lord CJ, Ashworth A. BRCAness revisited. Nat Rev Cancer. 2016;16:110-20.

2. Kadouri L, Hubert A, Rotenberg Y, et al. Cancer risks in carriers of the BRCA1/2 Ashkenazi founder mutations. J Med Genet. 2007;44:467-71.

3. Kirchhoff T, Satagopan JM, Kauff ND, et al. Frequency of BRCA1 and BRCA2 mutations in unselected Ashkenazi Jewish patients with colorectal cancer. J Natl Cancer Inst. 2004;96:68-70.

4. Niell BL, Rennert G, Bonner JD, Almog R, Tomsho LP, Gruber $\mathrm{SB}$. BRCA1 and BRCA2 founder mutations and the risk of colorectal cancer. J Natl Cancer Inst. 2004;96:15-21.

5. van Asperen CJ, Brohet RM, Meijers-Heijboer EJ, et al. Cancer risks in BRCA2 families: estimates for sites other than breast and ovary. J Med Genet. 2005;42:711-9.

6. Phelan CM, Iqbal J, Lynch HT, et al. Incidence of colorectal cancer in BRCA1 and BRCA2 mutation carriers: results from a follow-up study. Br J Cancer. 2014;110:530-4.

7. TCGA. Comprehensive molecular characterization of human colon and rectal cancer. Nature. 2012;487:330-7.
8. Parker JS, Mullins M, Cheang MC, et al. Supervised risk predictor of breast cancer based on intrinsic subtypes. J Clin Oncol. 2009;27:1160-7.

9. Prat A, Parker JS, Karginova O, et al. Phenotypic and molecular characterization of the claudin-low intrinsic subtype of breast cancer. Breast Cancer Res. 2010;12:R68.

10. Creighton CJ, Li X, Landis M, et al. Residual breast cancers after conventional therapy display mesenchymal as well as tumorinitiating features. Proc Natl Acad Sci USA. 2009;106:13820-5.

11. Goyal A, Terry MB, Jin Z, Siegel AB. C-reactive protein and colorectal cancer mortality in U.S. adults. Cancer Epidemiol Biomark Prev. 2014;23:1609-18.

12. Guinney J, Dienstmann R, Wang X, et al. The consensus molecular subtypes of colorectal cancer. Nat Med. 2015;21:1350-6.

13. Hugen N, Brown G, Glynne-Jones R, de Wilt JH, Nagtegaal ID. Advances in the care of patients with mucinous colorectal cancer. Nat Rev Clin Oncol. 2016;13:361-9.

14. Chen J, Pei Z, Dai L, et al. Transcriptome profiling using pyrosequencing shows genes associated with bast fiber development in ramie (Boehmeria nivea L.). BMC Genomics. 2014;15:919.

15. Larsen MJ, Kruse TA, Tan Q, et al. Classifications within molecular subtypes enables identification of BRCA1/BRCA2 mutation carriers by RNA tumor profiling. PLoS ONE. 2013;8:e64268.

16. Siegel R, Desantis C, Jemal A. Colorectal cancer statistics, 2014. CA Cancer J Clin. 2014;64:104-17.

17. Locker GY, Lynch HT. Genetic factors and colorectal cancer in Ashkenazi Jews. Fam Cancer. 2004;3:215-21.

18. Yurgelun MB, Kulke MH, Fuchs CS, et al. Cancer susceptibility gene mutations in individuals with colorectal cancer. J Clin Oncol. 2017;35:1086-895.

19. Murphy G, Devesa SS, Cross AJ, Inskip PD, McGlynn KA, Cook MB. Sex disparities in colorectal cancer incidence by anatomic subsite, race and age. Int J Cancer. 2011;128:1668-75.

20. Lin JH, Zhang SM, Rexrode KM, et al. Association between sex hormones and colorectal cancer risk in men and women. Clin gastroenterol hepatol. 2013;11:e1.

21. Negri FV, Azzoni C, Bottarelli L, et al. Thymidylate synthase, topoisomerase-1 and microsatellite instability: relationship with outcome in mucinous colorectal cancer treated with fluorouracil. Anticancer Res. 2013;33:4611-7.

22. Dienstmann R, Vermeulen L, Guinney J, Kopetz S, Tejpar S, Tabernero J. Consensus molecular subtypes and the evolution of precision medicine in colorectal cancer. Nat Rev Cancer. 2017;17: 79-92.

23. Ciriello G, Miller ML, Aksoy BA, Senbabaoglu Y, Schultz N, Sander C. Emerging landscape of oncogenic signatures across human cancers. Nat Genet. 2013;45:1127-233.

24. Narod SA. Earlier age of onset in BRCA carriers-anticipation or cohort effect?: a countercurrents series. Curr Oncol. 2011;18: 257-8.

25. https://seer.cancer.gov/statfacts/html/anus.html. (Accessed Jan 2017). 